\title{
Determination of prestenotic flow volume using an automated method based on colour Doppler imaging for evaluating orifice area by the continuity equation: validation in a pulsatile flow model
}

\author{
K Dennig, H J Nesser, D Hall, H U Haase, A Schömig
}

\begin{abstract}
Objective-To evaluate, in a pulsatile flow model simulating flow conditions in valvar stenoses, whether accurate determination of orifice area can be achieved by the continuity equation using automated determination of flow volumes based on spatiotemporal integration of digital colour Doppler flow velocities.
\end{abstract}

Methods-A method for automated determination of flow volumes which takes into account the velocity distribution across a region of interest was examined using flow through a tube and various restrictive outlet orifices with areas ranging between 0.2 and $3.1 \mathrm{~cm}^{2}$. The sampling rectangle of the Doppler method was positioned proximal to the obstructions within the flow convergence zone for evaluating prestenotic flow volume. Stenotic jet velocities were recorded by continuous wave Doppler to obtain the integral under the velocity curve. Prestenotic flow volume was then divided by the velocity integral to calculate functional orifice area according to the continuity equation.

Results-The presence of parabolically shaped velocity profiles across the prestenotic region was demonstrated by the Doppler method. Excellent agreement was found between prestenotic flow volumes measured by the Doppler technique and actual values $(r=0.99, \mathrm{SEE}=1.35 \mathrm{ml}$, $y=0.99 x-0.24)$. Use of the continuity equation led to a close correlation, with a systematic underestimation of geometric orifice sizes. Correction of Doppler data for flow contraction yielded an excellent agreement with actual orifice areas.

Conclusions-The study validated the accuracy of a Doppler method for automated determination of flow volumes for quantifying orifice area by the continuity equation. Prestenotic flow volume and functional orifice area could be evaluated reliably in the presence of non-flat velocity profiles. Thus the method contributes to the non-invasive assessment of valvar stenoses.

(Heart 1998;79:324-330)

Keywords: Doppler echocardiography; automated flow volume determination; valvar stenoses; pulsatile flow model
Clinical studies in valvar stenoses have shown the diagnostic value of orifice area determination using the continuity equation based on Doppler echocardiographic measurements. ${ }^{1-9}$ It was recognised that there were potential shortcomings over the measurement of the variables necessary for the continuity equation. In particular, the assumption of a flat velocity profile across the prestenotic region is of critical importance, since prestenotic flow is usually evaluated by pulsed Doppler recordings of the velocity within the centre of the flow area. Flow acceleration close to the stenotic valve and subvalvar obstruction due to extensive calcification or septal hypertrophy may preclude a flat profile. A further requirement-namely that the echocardiographically measured geometrical dimensions of the valvar rings are equal to prestenotic flow area, which remains constant during the flow period-is also not optimally fulfilled. A method for determining flow volume which takes into account the velocity distribution across a flow region of interest and its variation during the flow interval may improve the evaluation of orifice area by the continuity equation since it is less dependent on these simplifying assumptions.

The purpose of this study was to evaluate, in a pulsatile flow model simulating flow conditions in valvar stenoses, whether accurate determination of orifice area by the continuity equation can be achieved, using a recently developed method for automated determination of flow volumes based on spatiotemporal integration of digital colour Doppler flow velocities. In particular, we addressed the question of whether measurements by this new method would allow us to evaluate actual prestenotic flow volume within the flow convergence zone close to an obstruction, where a non-flat velocity profile is present.

\section{Methods}

PULSATILE FLOW MODEL

The pulsatile flow model consisted of a closed circulation with tubes mountable into the circuit (fig 1). At the outlet of a silicone tube, the cross sectional area could be reduced by insertion of plates with various orifices. A computer adjusted pump provided flow volumes and pump rates with various programmable waveforms. The flow volumes were cross checked by an ultrasonic transit time flowmeter (T108, Transonic Systems, New York, USA). For all measurements, a glycerol-water solution $(40: 60)$ was used as test fluid with a physiological dynamic 


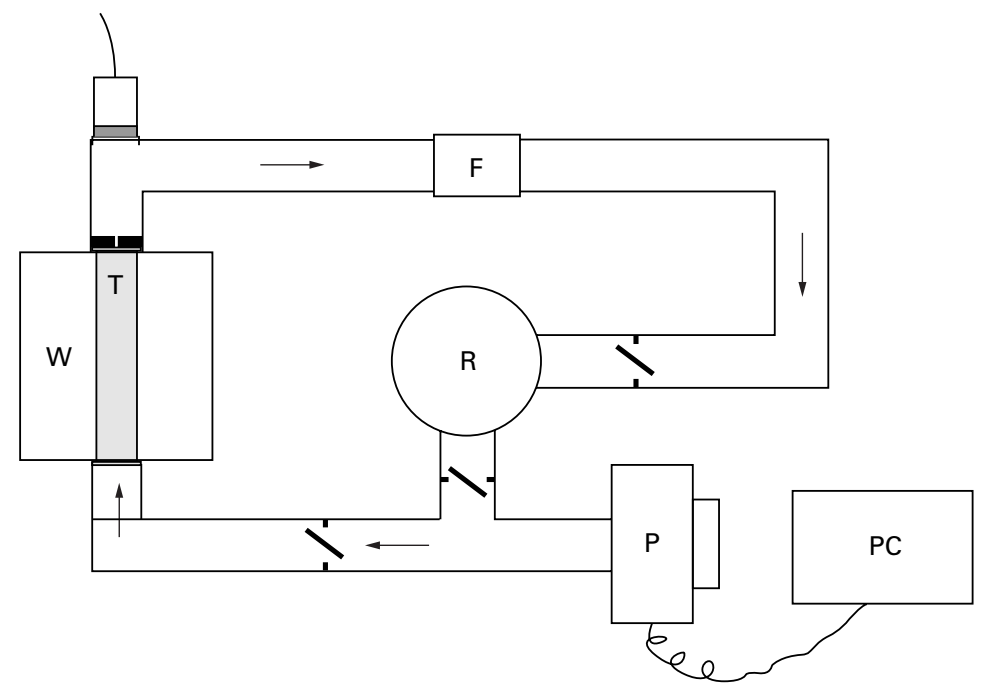

Figure 1 Schematic drawing of the pulsatile flow model. A computer (PC) controlled pump $(P)$ cross checked by a flow meter $(F)$ delivers flow volumes from a fluid reservoir (R) into a closed circulation. The test tube (T), at the outflow region of which various restrictive orifices can be inserted, is surrounded by a water tank (W) to improve ultrasound imaging conditions. Ultrasound recordings can be performed through a membrane against flow direction from the upper transducer position.

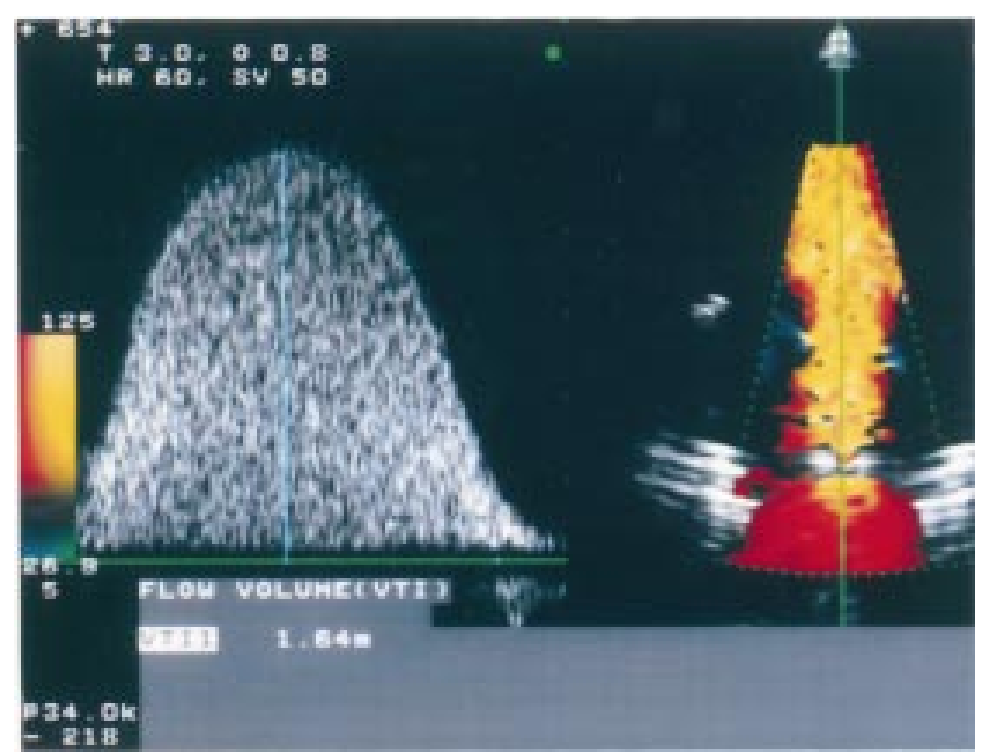

Figure 2 The colour Doppler image on the right side shows a jet towards the transducer created by the insertion of a circular shaped orifice with a diameter of $0.8 \mathrm{~cm}$ and the orientation of the $C W$ Doppler beam through the centre of the jet. On the left side are shown the corresponding temporal velocity profile resembling that of stenotic jets at the semilunar valves and the calculated integral under the velocity curve.

viscosity of $3.6 \mathrm{cP}$. For improved echo reflectivity $1 \%$ cornstarch particles were added.

DOPPLER SYSTEM AND PRINCIPLE OF FLOW VOLUME COMPUTATION

Colour Doppler imaging was performed with a Toshiba SSA-380A Doppler system (Toshiba, Tokyo, Japan) equipped with a recently developed software for automated evaluation of stroke volume and cardiac output based on digital data of velocity recordings. ${ }^{10}$ The method allows selection of an optimal flow cycle from cine-loop stored colour Doppler images. For further calculation, the flow interval is then given by the time delay between the first and the last depicted frame. To ensure sufficient temporal resolution capabilities, high frame rates are available. A review of the entire selected cycle in real time is provided by the Doppler system.

Automated determination of flow volumes is based on the analysis of the velocity distribution across a region of interest within the colour coded image. The computational algorithm uses the digital velocities of the Doppler flow map and the system shows a sampling rectangle which can be positioned across the flow field of interest perpendicular to the central Doppler beam direction. Additionally, correction for angle deviations between flow direction and the orientation of the sampling rectangle is provided. The size of the sampling rectangle depends on the imaging depth $\mathrm{z}$ (in $\mathrm{cm}$ ) used and measures $0.285 \mathrm{z}$ horizontally and $0.0385 \mathrm{z}$ vertically. The velocity distribution is delineated across five equidistant horizontal lines traversing the sampling box. For each frame, analysis of the velocity profiles $\mathrm{v}(\mathrm{r})$ (whereby $\mathrm{v}(\mathrm{r}$ ) denotes the velocity at a distance $r$ from the centre) across the five sampling lines is performed and the profile of the middle line is subsequently shown on the screen for each analysed frame. The integral under the velocity curve is approximated by summation over the velocity-radius rectangles, $\sum \mathrm{v}(\mathrm{r}) \Delta \mathrm{r}$, where $\Delta \mathrm{r}$ denotes the distance between two neighbouring scanning lines. Rotational spatial integration of the velocity areas, assuming a circular shaped flow area and hemicircular identity of the spatial velocity distribution, is carried out for each point in time during the flow interval, yielding instantaneous flow rates, $\pi \sum \mathrm{r} v(\mathrm{r}) \Delta \mathrm{r}$. Temporal summation of the flow rates over the number of registered flow frames through the entire flow interval, $\pi \sum \sum \mathrm{r}(\mathrm{t}) \mathrm{v}(\mathrm{r}, \mathrm{t}) \Delta \mathrm{r} \Delta \mathrm{t}(\Delta \mathrm{t}$ denotes the constant time interval between two subsequent frames, which depends on the applied colour Doppler frame rate), is then performed, yielding five distinct flow volumes from which the mean value and standard deviation are calculated and depicted on the screen.

\section{DATA MEASUREMENTS}

A fixed pump rate of 60 strokes/min was used for all recordings, and a sinusoidal waveform for the forward flow curves simulating ventricular outflow conditions was chosen (fig 2). Flow volumes between 20 and $90 \mathrm{ml}$ (for the smallest orifice size up to $70 \mathrm{ml}$ ), varied in $10 \mathrm{ml}$ steps, were delivered by the pump into the circulation. Measurements were performed at the outlet region of a silicone tube with a diameter of $3 \mathrm{~cm}$ and a length of $14 \mathrm{~cm}$. Five different plates with sharp edged circular orifices with diameters of $0.5,0.8,1.0,1.5$, and $2.0 \mathrm{~cm}$ (orifice areas of $0.2,0.5,0.8,1.8$, and $3.1 \mathrm{~cm}^{2}$ ) were positioned into the outlet.

For all experiments, a transducer carrier frequency of $3.75 \mathrm{MHz}$ was employed. The Doppler instrumentation settings were adjusted to the corresponding flow conditions with a pulse repetition frequency of $6.0 \mathrm{kHz}$ and zero line shifting leaving about $75 \%$ of the velocity range for colour coded flow towards the transducer such that no aliasing occurred. Wall filtering was minimised and ranged between 76 and $609 \mathrm{~Hz}$ for the applied Nyquist velocities between 9.49 and $75.9 \mathrm{~cm} / \mathrm{s}$. Gain settings were 


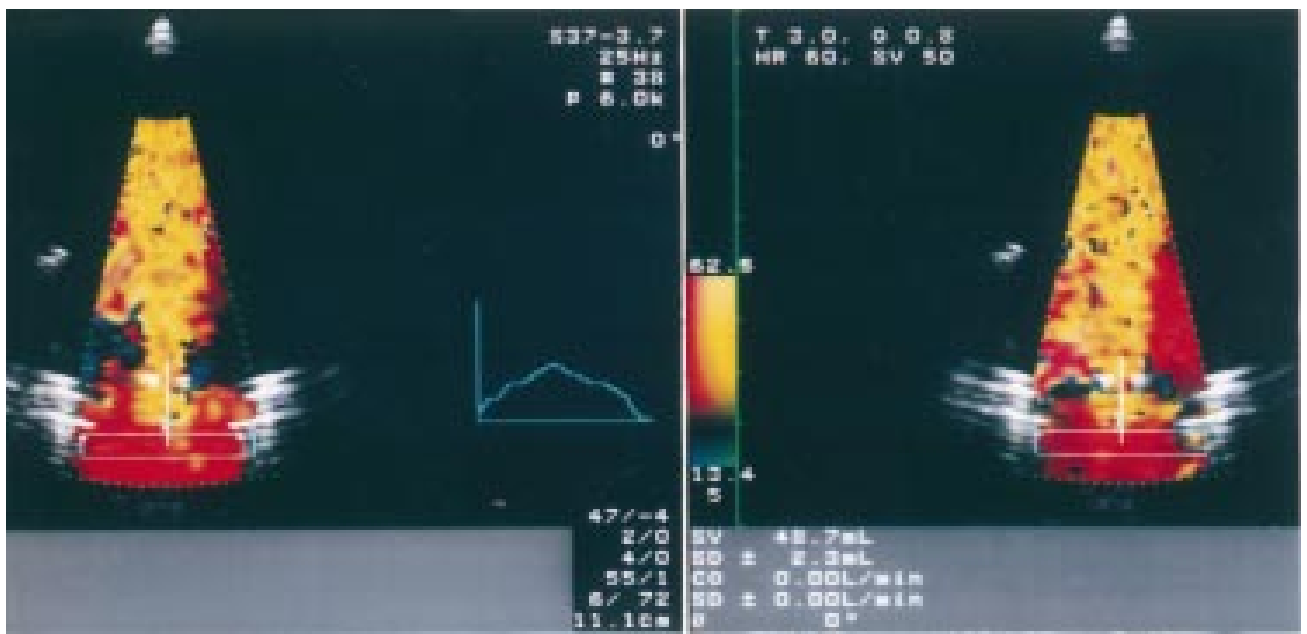

Figure 3 Example of the application of the algorithm for automated determination of prestenotic flow volume from colour Doppler imaging using a volume of $50 \mathrm{ml}$ through an orifice with a diameter of $0.8 \mathrm{~cm}$. Flow toward the transducer, colour coded in red, is shown on the left panel with increasing flow velocities within the centre of the flow convergence zone close to the obstruction. The sampling rectangle of the automated method is positioned into the flow convergence zone and an instantaneous velocity profile across its diameter is depicted on the right sided graph as a parabolically shaped spatial profile. The panel on the right side shows the last colour Doppler image of the chosen flow interval and the calculated flow volume is depicted within the bar on the bottom of the image.

adjusted to the various flow conditions just below the level where noise in the colour Doppler image occurred. The colour sector size was set to $26^{\circ}$. Using these machine settings frame rates between $24 / \mathrm{s}$ and $26 / \mathrm{s}$ were derived.

The transducer was located and fixed $8 \mathrm{~cm}$ away from the orifices at a specially constructed window in the model, and the central Doppler beam was oriented parallel to and against flow direction. Accordingly, for all experiments, the angle between flow direction and the orientation of the sampling rectangle was set to zero since flow direction was paralleled by Doppler interrogation. For colour Doppler imaging a constant depth of $12.0 \mathrm{~cm}$ was applied; thus a sampling box with a lateral width of $3.462 \mathrm{~cm}$ and a vertical depth of $0.462 \mathrm{~cm}$ was used. The sampling rectangle was positioned $0.5 \mathrm{~cm}$ proximal to the orifice for determination of prestenotic flow volume $\left(F_{\mathrm{D}}\right)$. From the identical transducer position and orientation, stenotic jet velocity through the orifices was recorded by continuous wave Doppler, and using a hand controlled cross wire cursor the Doppler spectral curve was traced along its envelope to obtain the integral under the profile (Int v). Stenotic orifice area $\left(\mathrm{OA}_{\mathrm{D}}\right)$ was then calculated by the continuity equation according to $\mathrm{OA}_{\mathrm{D}}=\mathrm{FV}_{\mathrm{D}} /$ Int v. Since effective orifice areas were determined by the continuity equation, these data were also multiplied by the correction factor $1 / 0.65$ for flow contraction behind an abrupt obstruction to derive geometric orifice areas. ${ }^{11}$ For every discrete combination of flow rate and orifice size, a sequence of five colour Doppler flow cycles, as well as CW Doppler recordings through the centre of the orifices, was acquired on the ultrasound scanner.

STATISTICAL ANALYSIS

All measurements were averaged over five cycles and results expressed as mean (SD). Actual and measured flow volumes, as well as calculated and true orifice areas, were compared by linear regression analysis. A $\mathrm{p}$ value $<0.05$ was considered statistically significant. The repeatability of measurements was expressed as two standard deviations from the mean difference of five measurements for each orifice under various flow conditions according to the method of Bland and Altman. ${ }^{12}$

\section{Results}

FLOW VOLUMES AND VELOCITIES

Analysis of the velocity distribution by the algorithm showed the presence of parabolically shaped profiles across the prestenotic flow region (fig 3). Flow volumes evaluated by the Doppler method averaged over all orifice sizes measured 19.2 (0.8), 29.3 (1.8), 40.1 (1.4), 49.6 (1.6), 58.7 (2.1), 69.8 (2.0), 79.6 (3.0), and $87.6(2.3) \mathrm{ml}$ for actual flow volumes of between 20 and $90 \mathrm{ml}$. Peak velocities of stenotic jet flows ranged from 0.1 to $6.5 \mathrm{~m} / \mathrm{s}$ and the corresponding integrals under the velocity curves from 3 to $502 \mathrm{~cm}$ for the entire range of flow volumes and orifice sizes.

\section{COMPARISON OF FLOW VOLUMES AND ORIFICE} AREAS

The comparison of measured flow volumes derived by the Doppler technique and actual flow volumes showed an excellent correlation for each orifice size, with no relevant underestimation or overestimation (figs 4 and 5). For the entire range of measurements from all orifice sizes the overall agreement was characterized by: $r=0.99, \quad \mathrm{SEE}=1.35 \mathrm{ml}$, $y=0.99 x-0.24$. With the application of the continuity equation the calculated effective orifice areas under the applied flow conditions measured 0.14 (0.05), 0.32 (0.01), 0.51 (0.02), $1.08(0.03)$, and $2.15(0.03) \mathrm{cm}^{2}$ for the $0.2,0.5,0.8,1.8$, and $3.1 \mathrm{~cm}^{2}$ geometric orifice sizes, respectively. Thus the comparison of calculated and geometric orifice areas showed a close correlation with a systematic deviation 


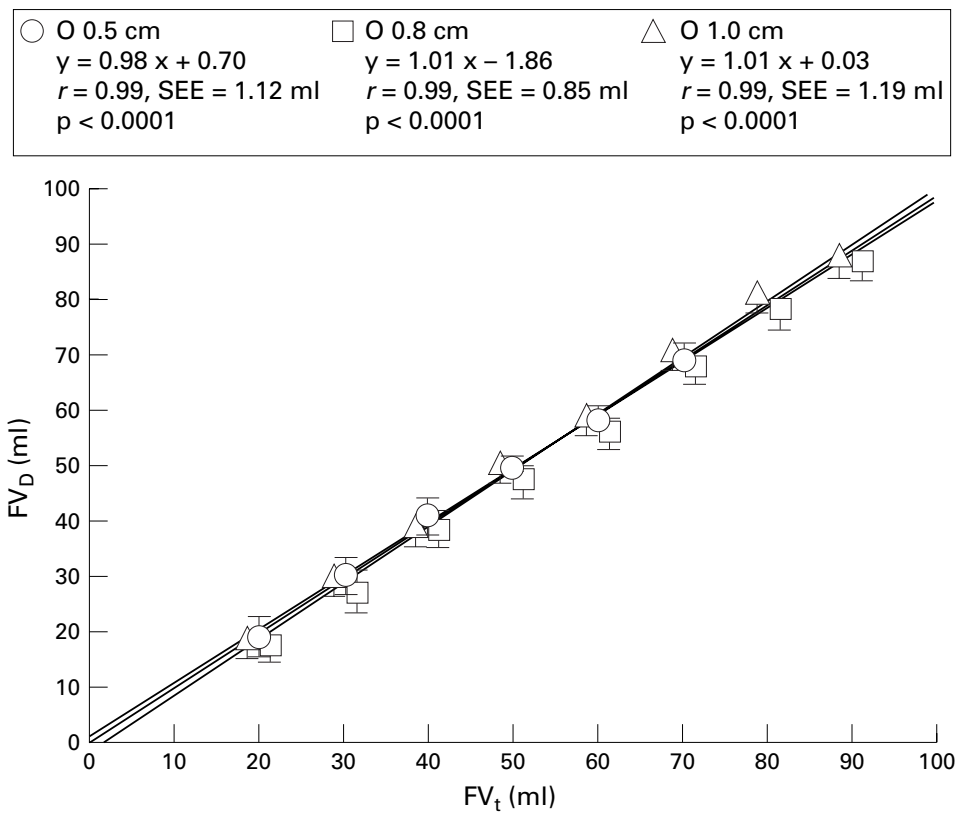

Figure 4 Correlation of true flow volumes $(F V)$ and measured flow volumes by the Doppler technique $\left(F V_{D}\right)$ in the prestenotic region for the smaller orifice sizes with a diameter of 0.5 (circles), 0.8 (squares), and $1.0 \mathrm{~cm}$ (triangles). Mean values and standard deviation from five measurements are depicted.

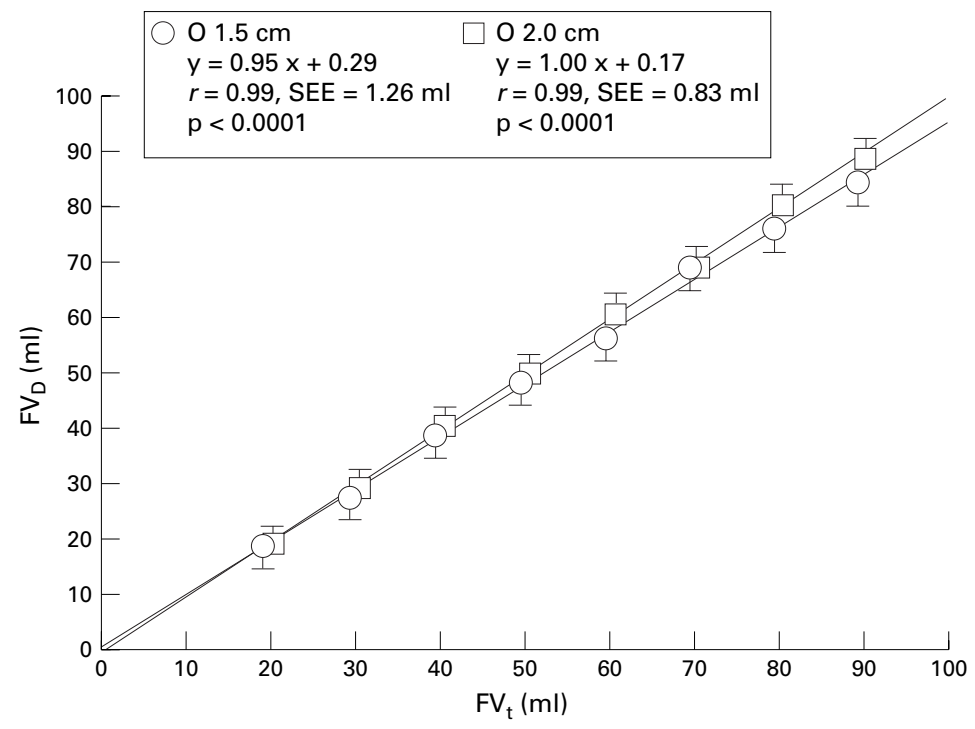

Figure 5 Measured flow volumes (ordinate) plotted against actual flow volumes (abscissa) for the larger orifice sizes with a diameter of 1.5 (circles) and $2.0 \mathrm{~cm}$ (squares). Mean values and standard deviations from five measurements are shown.

of the regression line from the line of identity $\left(r=0.99, \operatorname{SEE}=0.06 \mathrm{~cm}^{2}\right.$, $\mathrm{y}=0.68 \mathrm{x}-0.03$ ) (fig 6). Multiplying the functional areas with the correction factor of 1.54 for flow contraction a close agreement of Doppler data with actual geometric orifice areas was found $\left(r=0.99, \operatorname{SEE}=0.06 \mathrm{~cm}^{2}\right.$, $\mathrm{y}=1.05 \mathrm{x}-0.03)($ fig 6$)$.

MEASUREMENT REPEATABILITY

There was excellent agreement between repeated measurements: two residual standard deviations measured $2.4 \mathrm{ml}$ for the circular orifice with a diameter of $0.5 \mathrm{~cm}, 3.2 \mathrm{ml}$ for $0.8 \mathrm{~cm}, 4.8 \mathrm{ml}$ for $1.0 \mathrm{~cm}, 2.9 \mathrm{ml}$ for $1.5 \mathrm{~cm}$, and $3.9 \mathrm{ml}$ for $2.0 \mathrm{~cm}$ for the applied range of flow conditions.

\section{Discussion}

The major finding of this in vitro study was that with the use of a recently developed method for automated determination of flow volumes based on colour Doppler imaging, quantification of orifice area according to the continuity equation can be achieved, since it allows reliable evaluation of prestenotic flow volume and obviates the need for various simplifying assumptions. The approach takes into account the non-flat velocity profile within the flow convergence region of the stenoses. In addition, the applied approach may be less time consuming than the conventional application of the continuity principle based on echocardiographic and Doppler measurements. The calculated orifice areas showed a close agreement with the effective orifice areas and, since the flow conditions employed were similar to those in valvar stenoses, the method should lead to substantial improvement in the quantitative assessment of these lesions. In contrast to clinical studies, where the underestimation of geometric aortic and mitral valve areas reaches only about $15 \%$, the underestimation in our study was more pronouncedand thus the correction factor for flow contraction was substantially higher-because the abrupt obstructions used in our study resemble an ideal nozzle far less than valvar stenoses. ${ }^{124891314}$

\section{VALVAR RING VELOCITY PROFILES IN NORMAL} SUBJECTS

Even in normal hearts the velocity profile in the left ventricular outflow tract has been shown to be non-flat, with the highest velocities occurring along the interventricular septum. ${ }^{15} 16$ Though such a skewed profile is present in healthy persons, the velocity measured centrally within the outflow tract renders an acceptable approximation of mean spatial velocity since the velocity values decrease continuously towards the anterior mitral leaflet. Because of this, numerous studies using the centrally recorded profiles by pulsed Doppler, and taking into consideration the corresponding area measured by echocardiography, have shown close agreement with actual stroke volume through the left ventricular outflow tract. ${ }^{17-23}$ At the mitral ring level, a non-flat profile was found even with normal valves and the centrally measured velocity is often not representative of mean spatial velocity. ${ }^{24}$

\section{PRESTENOTIC ANNULAR VELOCITY PROFILES}

The clinical use of the continuity equation for determining orifice area in valvar stenosis, in particular at the aortic valve, is often limited by the presence of a non-flat velocity profile in the prestenotic region. ${ }^{25-27}$ In valvar stenosis, an increase in flow velocity occurs in the proximity of the valve, with the highest velocities located in the centre of the cross section due to flow convergence. To avoid overestimation of prestenotic flow, the sample volume of pulsed Doppler must be located proximal to this region. ${ }^{9}$ This approach may result in recordings being made at a site substantially different from that at which the diameter of flow area is 


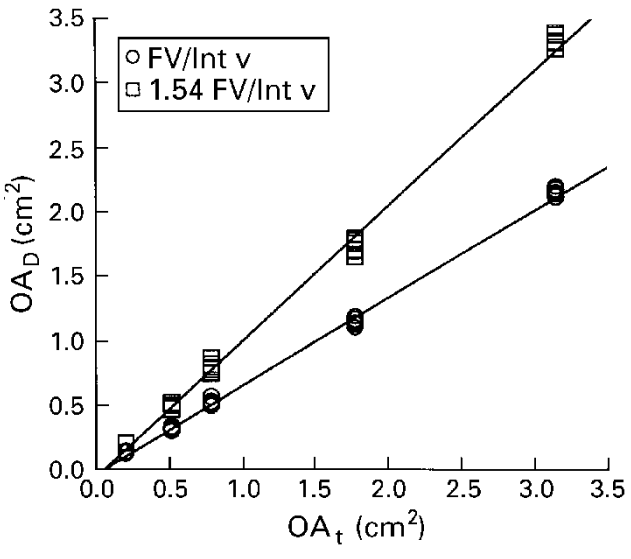

Figure 6 Relation between true geometric orifice areas $(O A)$ and orifice areas calculated by the application of the continuity equation $\left(O A_{D}\right)$. Functional orifice areas (circles) determined from the Doppler parameters are closely correlated and systematically smaller than geometric areas. Correction of Doppler data (squares) for flow contraction behind the abrupt obstructions by the factor of 1.54 yielded a close agreement with actual orifice sizes.

determined. The presence of marked leaflet calcification and septal hypertrophy in aortic stenosis may also prevent the occurrence of a symmetrical velocity profile. Further alterations in the spatial velocity profile at the mitral ring occur if annular calcification is present. Therefore in many clinical situations velocity measurements in the centre of the flow area that are based on the assumption of a flat velocity profile within the prestenotic region of the valve may lead to substantial errors in determining flow volume. Our data show that the new Doppler method allows reliable assessment of prestenotic flow in the flow convergence zone of an obstruction. The velocity profiles across this region were found to be parabolically shaped, with the highest velocities in the centre. Since the applied algorithm for flow determination takes into account the velocity distribution across a region of interest and not just the central velocity, no significant overestimation was found.

TEMPORAL ALTERATIONS OF FLOW AREA SIZE

With the conventional employment of the continuity equation, the diameter of flow area is usually evaluated at a particular time point, assuming that the measured diameter and calculated area remain constant during the entire flow interval. Even though this represents an acceptable approximation for the aortic ring, substantial changes in mitral ring size can occur during diastole. It has been shown that the ring area of the mitral valve can vary by $26 \%$ during the cardiac cycle and by approximately $13 \%$ in diastole in healthy persons. ${ }^{28}$ Accordingly, measuring the diameter of the flow area at a single time point during the flow cycle may lead to deviations of the calculated prestenotic flow and thus of orifice area. The Doppler method investigated here takes into consideration the changes of the dimension of the flow area during the flow period since an instantaneous diameter is determined from each colour Doppler image.
TIME AND EFFORT FOR APPLICATION

Clinical employment of the conventional method of orifice area determination by the continuity equation is time consuming since it requires accurate measurements of three distinct variables. In aortic stenosis, the dimension of the aortic ring is usually evaluated from a parasternal view, while prestenotic and stenotic jet velocities are recorded from an apical transducer position. Most investigators used the peak velocities of the prestenotic and stenotic jet flow velocity profiles, since time is not required to trace the velocity curves. Since with the use of this new method, prestenotic flow volume-usually derived from two separate measurements - can be determined within less than one minute from a single transducer position, the time needed for accurate measurement can be reduced.

STUDY LIMITATIONS

Since this Doppler method uses the velocity information from cross sectional images, a potential source of error may result from the underlying assumption of a hemicircular symmetry of the spatial velocity profile. This may be specially relevant if skewed profiles are present because of septal hypertrophy or marked valvar calcium deposits in aortic stenosis. This situation was not simulated in the study; however, even under those circumstances a more reliable estimation of prestenotic flow volume is likely to be derived by the new method than by measuring flow velocity at a single point in the centre of the flow region. The degree to which non-symmetrical spatial velocity profiles impair the accuracy of the method needs further clarification.

An additional simplification in comparison with the clinical situation results from the assumption of a circular shaped flow area with the new method. The assumption is best fulfilled at the aortic and pulmonary ring level; however, substantially different geometries may be present at the mitral and the tricuspid rings. ${ }^{28}{ }^{29}$ Thus the use of the method to take measurements across a single flow diameter may be restricted to the ventricular outflow tract regions. Though previous studies of mitral flow volume in normal individuals using a single diameter measurement showed a close agreement with invasive values, modifications of the method may be necessary to obtain a more accurate quantification of prestenotic flow. ${ }^{29}$

We also did not address the issue of angle deviations between flow direction and Doppler beam orientation. Theoretically, small angle deviations play a minor role in the accuracy of the method since the cosine function of the angle is involved in the determination of flow volumes. ${ }^{10}$ Accordingly, angle deviations of up to $20^{\circ}$ are only associated with calculation errors of up to $6 \%$. In addition, the algorithm allows for correction of misalignments using the cosine function of the angle between the central Doppler beam orientation and flow direction, as depicted by the operator with a moveable cursor. Since for large angles, however, Doppler recordings are impaired by a 
low signal to noise ratio, such angles will also impair the accuracy of flow volume measurements made by this method. Fortunately, in valvar stenosis small angles between prestenotic flow direction and Doppler beam orientation can usually be achieved by appropriate transducer positioning.

Finally, the potential influence of various machine settings such as gain and wall filtering was not investigated in this study. Corresponding to the clinical application, colour Doppler gain was adjusted to a level immediately below that at which noise is incurred, yielding excellent results in these in vitro experiments. The extent to which gain and wall filtering may affect the accuracy of the method was investigated in recent studies. ${ }^{31}$ Incomplete flow visualisation because of inadequate gain setting or attenuation of ultrasound was shown to be an important bar to reliable flow volume determination by the automated method ${ }^{31}$; in both the clinical and the in vitro part of this study, it was quite clear to the examiner when the colour gain was either insufficient or excessive for an optimal gain setting to be achieved. In an in vitro study, it was pointed out that attenuation effects were not negligible but were more pronounced if larger Doppler angles, lower flows, and high wall filter settings were employed, leading to various degrees of underestimation of flow calculations. ${ }^{30}$ Since no motion of the surrounding surfaces was present in our study, wall filters between 76 and $609 \mathrm{~Hz}$ were used. This may be too low a value for clinical application since strong reflectors such as surrounding walls in motion may influence the flow area image on colour Doppler and thus affect the calculated flow volume. The tissue priority algorithm of the instruments must also be taken into account for adequate imaging of the flow zone. Careful adjustment with the use of wall filtering and the motion artefact eliminator, which neglects strong and low velocity signals, may be necessary for reliable calculations.

CONCLUSIONS

Our data show the value of a new Doppler method for determining prestenotic flow volume, based on the spatial velocity distribution across the corresponding flow region for assessing orifice area with the continuity equation. The method, which takes into account instantaneous flow alterations by making determinations at several time points during the flow interval, should allow a more reliable measurement of flow volume in the clinical setting. Since rapid assessment of prestenotic flow volume can substantially shorten the time needed for accurate calculations, the method will improve the applicability of the continuity equation for assessing valvar stenoses.

1 Come PC, Riley MF, Ferguson JF, et al. Prediction of severity of aortic stenosis: accuracy of multiple noninvasive parameters. Am $\mathcal{F}$ Med 1988;85:29-37.

2 Dennig K, Kraus F, Rudolph W. Doppler echocardiographic determination of the orifice area in aortic valve stenosis determination of the orifice area in aortic valve stend
using the continuity equation. Herz 1986;11:309-17.

3 Derumeaux G, Bonnemains T, Remadi F, et al. Noninvasive assessment of mitral stenosis before and after percutaneous balloon mitral valvotomy by Doppler continuity equation. Eur Heart $\mathcal{F}$ 1992;13:1034-9.
4 Grayburn PA, Smith MD, Harrison MR, et al. Pivotal role of aortic valve area calculation by the continuity equation for Doppler assessment of aortic stenosis in patients with combined aortic stenosis and regurgitation. Am 7 Cardiol 1988; 61:376-81.

5 Harrison MR, Gurley JC, Smith MD, et al. A practical application of Doppler echocardiography for the assessment of severity of aortic stenosis. Am Heart $\mathcal{F} 1988 ; 115$ : $622-8$.

6 Karp K, Teien D, Eriksson P. Doppler echocardiographic assessment of the valve area in patients with atrioventricular valve stenosis by application of the continuity equation. F Intern Med 1989;225:261-6.

7 Nakatani S, Masuyama T, Kodama K, et al. Value and limitations of Doppler echocardiography in the quantification of stenotic mitral valve area: comparison of the pressure half-time and the continuity equation methods. Circulation 1988;77:78-85.

8 Oh JK, Taliercio CP, Holmes DR, et al. Prediction of the severity of aortic stenosis by Doppler aortic valve area determination: prospective Doppler-catheterization correlation in 100 patients. F Am Coll Cardiol 1988;11: 1227-34.

9 Skjaerpe T, Hegrenaes L, Hatle L. Noninvasive estimation of valve area in patients with aortic stenosis by Doppler ultrasound and two-dimensional echocardiography. Circulation 1985;72:810-18.

10 Tsujino $\mathrm{H}$, Shiki E, Hirama $\mathrm{M}$, et al. Quantitative measurement of volume flow rate (cardiac output) by the multibeam Doppler method. F $\mathrm{Am}$ Soc Echocardiogr 1995;8:621-30.

11 Blevins RD. Applied fluid dynamics handbook. New York: Van Nostrand, 1984:140.

12 Altman DG, Bland JM. Measurement in medicine: the analysis of method comparison studies. Statistician 1983; 32:307-17.

13 Teirstein P, Yeager M, Yock PG, et al. Doppler echocardiographic measurement of aortic valve area in aortic stenosis: a noninvasive application of the Gorlin formula. $7 \mathrm{Am}$ Coll Cardiol 1986;8:1059-65.

14 Zoghbi WA, Farmer KL, Soto JG, et al. Accurate noninvasive quantification of stenotic aortic valve area by Doppler echocardiography. Circulation 1986;73:452-9.

15 Kupari M, Koskinen P. Systolic flow velocity profile in the left ventricular outflow tract in persons free of heart disease. Am f Cardiol 1993;72:1172-8.

16 Rossvoll O, Samstad S, Torp HG, et al. The velocity distribution in the aortic annulus in normal subjects: a quantitative analysis of two-dimensional Doppler flow maps. $\mathcal{f} \mathrm{Am}$ Soc Echocardiogr 1991;4:367-78.

17 Dittmann H, Voelker W, Karsch K-R, et al. Influence of sampling site and flow area on cardiac output measurements by Doppler

18 Ihlen H, Pamlie JP, Dale J, et al. Determination of cardiac output by Doppler echocardiography. Br Heart $f$ 1984;51: 54-60.

19 Labovitz AJ, Buckingham TA, Habermehl K, et al. The effect of sampling site on the two-dimensional echoDoppler determination of cardiac output. Am Heart $\mathcal{f}$ 1985;109:327-32.

20 Lewis JF, Kuo LC, Nelson JG, et al. Pulsed Doppler echocardiographic determination of stroke volume and cardiac output: clinical validation of two new methods using the apical window. Circulation 1984;70:425-31.

21 Loeppky JA, Hoekenga DE, Greene R, et al. Comparison of noninvasive pulsed Doppler and Fick measurements of noninvasive pulsed Doppler and Fick measurements of
stroke volume in cardiac patients. Am Heart $\mathcal{F} 1984 ; 107$ : 339-46.

22 Nicolosi GL, Pungercic E, Cervesato E, et al. Feasibility and variability of six methods for the echocardiographic and Doppler determination of cardiac output. Br Heart f 1988; 59:299-303

23 Stewart WJ, Jiang L, Mich R, et al. Variable effects of changes in flow rate through the aortic, pulmonary and mitral valves on valve area and flow velocity: impact on quantitative Doppler flow calculations. $\mathcal{F} \mathrm{Am}$ Coll Cardiol 1985;6:653-62.

24 Samstad SO, Torp HG, Linker DT, et al. Cross sectional early mitral flow velocity profiles from colour Doppler. $\mathrm{Br}$ Heart f 1989;62:177-84.

25 Samstad SO, Rossvoll O, Torp HG, et al. Cross-sectional early mitral flow-velocity profiles from color Doppler in patients with mitral valve disease. Circulation 1992;86:74855.

26 Wiseth R, Samstad S, Rossvoll O, et al. Cross-sectional left ventricular outflow tract velocities before and after aortic valve replacement: a comparative study with twodimensional Doppler ultrasound. F Am Soc Echocardiogr 1993;6:279-85.

27 Zhou YQ, Faerestrand S, Matre K. Velocity distributions in the left ventricular outflow tract in patients with valvular aortic stenosis. Effect on the measurement of aortic valve area by using the continuity equation. Eur Heart f 1995;16: 383-93.

28 Ormiston JA, Shah PM, Tei C, et al. Size and motion of the mitral valve annulus in man. I. A two-dimensional echocardiographic method and findings in normal subjects. Circulation 1981;64:113-20.

$29 \mathrm{Pu} \mathrm{M}$, Griffin BP, Vandervoort PM, et al. Intraoperative validation of mitral inflow determination by transesophageal echocardiography: comparison of single-plane, biplane and thermodilution techniques. $7 \mathrm{Am}$ Coll Cardiol 1995;26: 1047-53. 
30 Sun JP, Pu M, Fouad FM, et al. Automated cardiac output measurement by spatiotemporal integration of color data. In vitro and clinical validation. Circulation 1997;95:932-9.
31 Ogata T, Reid CL, Joung K, et al. Effect of Doppler angle, wall filter and tissue attenuation on automated calculation [apsing method: an in vitro study [abstract]. Circulation 1996;94:I-378.

\section{STAMPS IN CARDIOLOGY}

\section{Cardiopulmonary resuscitation}

Cardiopulmonary resuscitation has featured rarely on postage stamps. Some of the elements of life support and resuscitation are illustrated on the stamps from Great Britain, Gilbert and Ellice Islands, and Somalia. Great Britain issued a set of four stamps (for expedition, skills, service, and recreation) on 12 August 1981 to commemorate the 25th anniversary of the Duke of Edinburgh award scheme. Printed on phosphorised paper and designed by P Sharland, the 22 pence stamp for service (as part of the gold award) depicts bystander external cardiac massage. Gutter pairs of the stamp are shown. (All modern Great Britain commemoratives are printed in sheets of two panes separated by a blank margin called a gutter. This format first appeared in 1972 and the term gutter pair refers to two stamps separated by the gutter margin. Stamps in this format are obviously rarer than singles.) Gilbert and Ellice Islands in the South Pacific issued three stamps in 1970 to commemorate the centenary of the British Red Cross. The International Red Cross was founded in 1863 following proposals by Jean-Henri Dunant (1828-1910), a Swiss businessman who witnessed the carnage at the Battle of Solferino on 24 June 1859. Appalled at the lack of medical aid and facilities for the wounded he published his book $A$ memory of Solferino and circulated it to European heads of government advocating that neutral status should be given to the wounded and the facilities used to transport and treat them. On 26 October 1863 a conference was held in Geneva where many of his proposals were accepted. On 22 August 1864 the Geneva Convention for the Amelioration of the Condition of the Wounded of Armies in the Field was signed. An insignia was required to identify these personnel and services, and the 1864 convention chose a red cross on a white field - the Swiss flag in reverse. The 10 cent Red Cross stamp from Gilbert and Ellice Islands shows mouth-to-mouth resuscitation. The only stamp issued that shows defibrillation is the 1980 stamp from Somalia as part of a set of three commemorating the 20th anniversary of the World Health Organisation whose logo is incorporated in the design. The stamp is listed in the Stanley Gibbons 1990 catalogue as representing "heart examination".

M K DAVIES A HOLLMAN 